Pacific

Journal of

Mathematics

HYPERSURFACES WITH CONSTANT CURVATURE QUOTIENTS IN WARPED PRODUCT MANIFOLDS

JiE WU AND ChaO XIA 


\title{
HYPERSURFACES WITH CONSTANT CURVATURE QUOTIENTS IN WARPED PRODUCT MANIFOLDS
}

\author{
JiE WU AND CHAO XiA
}

\begin{abstract}
We study rigidity problems for hypersurfaces with constant curvature quotients $\mathscr{H}_{2 k+1} / \mathscr{H}_{2 k}$ in the warped product manifolds. Here $\mathscr{H}_{2 k}$ is the $k$-th Gauss-Bonnet curvature and $\mathscr{H}_{2 k+1}$ arises from the first variation of the total integration of $\mathscr{H}_{2 k}$. Hence the quotients considered here are in general different from $\sigma_{2 k+1} / \sigma_{2 k}$, where $\sigma_{k}$ are the usual mean curvatures. We prove several rigidity and Bernstein-type results for compact or noncompact hypersurfaces corresponding to such quotients.
\end{abstract}

\section{Introduction}

Let $\Sigma^{n-1}$ be a closed smooth hypersurface isometrically immersed in an $n$-dimensional Riemannian manifold $\left(M^{n}, g\right)$. Assume that $\Sigma_{t}$ is a variation of $\Sigma$ with the unit outward normal vector field $v_{t}$ as the variational vector field. It is well known that the first variation of the area functional $\operatorname{Area}\left(\Sigma_{t}\right)$ is given by

$$
\left.\frac{d}{d t}\right|_{t=0} \operatorname{Area}\left(\Sigma_{t}\right)=\int_{\Sigma} H d \mu,
$$

where $H$ is the mean curvature of $\Sigma$ with respect to the inner normal and $d \mu$ is the area element of $\Sigma$. On the other hand, it is well known that the first variation of the total scalar curvature functional $\int_{\Sigma} R d \mu$ is given by

$$
\left.\frac{d}{d t}\right|_{t=0} \int_{\Sigma_{t}} R d \mu_{t}=\int_{\Sigma}-2 \sum_{i, j=1}^{n-1} E^{i j} h_{i j} d \mu,
$$

where $E^{i j}=R^{i j}-\frac{1}{2} R g^{i j}$ and $h_{i j}$ are respectively the Einstein tensor and the second fundamental form of $\Sigma$ with respect to the inner normal in the local coordinates.

There is a natural generalization of scalar curvature, called Gauss-Bonnet curvatures $L_{k}$ for an integer $1 \leq k \leq \frac{1}{2}(n-1)$ for $(n-1)$-dimensional Riemannian

Xia was supported by funding from the European Research Council under the European Union's Seventh Framework Programme (FP7/2007-2013) / ERC grant agreement no. 267087. Wu was supportec by the National Natural Science Foundation of China under Grant no. 11401553.

MSC2010: primary 53C24; secondary 52A20, 53C40.

Keywords: constant mean curvature, rigidity, warped product manifold, Gauss-Bonnet curvature. 
manifolds. $L_{k}$ are intrinsic curvature functions. When $n-1$ is even, the highest order Gauss-Bonnet curvature $L_{(n-1) / 2}$ is exactly the Pfaffian in the Gauss-BonnetChern formula. $L_{2}$ appeared first in [Lanczos 1938] and has been intensively studied in the theory of Gauss-Bonnet gravity, which is a generalization of Einstein gravity.

The first variation of the total Gauss-Bonnet curvature functional $\int_{\Sigma} L_{k} d \mu$ has been considered long time ago by Lovelock [1971]. Li [1985] also computed the first variation of these functionals as well as the second variation for submanifolds in the general ambient Riemannian manifolds. Recently an alternative computation was given by Labbi [2008b]:

$$
\left.\frac{d}{d t}\right|_{t=0} \int_{\Sigma_{t}} L_{k} d \mu_{t}=\int_{\Sigma}-2 \sum_{i, j=1}^{n-1} E_{(k)}^{i j} h_{i j} d \mu,
$$

where $E_{(k)}^{i j}$ is the generalized Einstein tensor defined by (2-1). Labbi [2008a] referred to the critical point of $\int_{\Sigma} L_{k} d \mu$ as $2 k$-minimal submanifolds. In this sense, the ordinary minimal submanifolds are referred as 0-minimal submanifolds.

For the ambient space $M^{n}=\mathbb{R}^{n}$, by the Gauss equation, one can verify that $L_{k}=(2 k) ! \sigma_{2 k}$ and $-2 \sum_{i, j=1}^{n-1} E_{(k)}^{i j} h_{i j}=(2 k+1) ! \sigma_{2 k+1}$, where $\sigma_{k}$ are the usual mean curvatures defined by the elementary symmetric functions of the principal curvatures of associated hypersurfaces. Hence the Gauss-Bonnet curvatures $L_{k}$ as well as the integrand $-2 \sum_{i, j=1}^{n-1} E_{(k)}^{i j} h_{i j}$ appear like higher order mean curvatures.

Throughout this paper, we use the notation

$$
\mathscr{H}_{2 k}:=L_{k}, \quad \mathcal{H}_{2 k+1}:=-2 \sum_{i, j=1}^{n-1} E_{(k)}^{i j} h_{i j},
$$

and call them $2 k$-mean curvature and $(2 k+1)$-mean curvature. By convention, we use $L_{0}=1$. We emphasize here that in general these mean curvatures are different from the usual ones defined by $\sigma_{k}$ except $\mathscr{H}_{0}$ and $\mathscr{H}_{1}$. The 0 -mean curvature $\mathscr{H}_{0}$ is equal to 1 and the 1-mean curvature $\mathscr{H}_{1}$ is equal to the usual mean curvature $H$.

We will consider some rigidity problems related to $\mathscr{H}_{2 k}$ and $\mathscr{H}_{2 k+1}$ in a class of Riemannian manifolds: warped product manifolds. A warped product manifold $(M, \bar{g})$ is the product manifold of an interval and an $(n-1)$-dimensional Riemannian manifold with some smooth positive warping function. Precisely,

$$
M=[0, \bar{r}) \times_{\lambda} N^{n-1} \quad(0<\bar{r} \leq \infty)
$$

is equipped with

$$
\bar{g}=d r^{2}+\lambda(r)^{2} g_{N},
$$

where $\lambda:[0, \bar{r}) \rightarrow \mathbb{R}_{+}$is a smooth positive function and $\left(N^{n-1}, g_{N}\right)$ is an $(n-1)$ dimensional Riemannian manifold. 
The rigidity problems for hypersurfaces in Riemannian manifolds with constant curvature functions are one of the central problems in the classical differential geometry. Historically, the rigidity problems for hypersurfaces in the Euclidean space was studied by Liebmann [1899], Hsiung [1954], Süss [1952], Alexandrov [1956; 1957; 1958a; 1958b], Alexandrov and Volkov [1958], Reilly [1977], Ros [1988], Korevaar [1988], etc. Recently, many works concerning the rigidity for hypersurfaces in warped product manifolds have appeared, see, for example, [Montiel 1999; Alías et al. 2013; Brendle 2013; Brendle and Eichmair 2013; Wu and Xia 2014] and the references therein.

In all above works, the curvature functions are related to the elementary symmetric functions $\sigma_{k}$ of the principal curvatures of hypersurfaces. Our concern in this paper is the curvature functions $\mathscr{H}_{2 k}$ and $\mathscr{H}_{2 k+1}$. In view of the Gauss equation, for hypersurfaces in general ambient Riemannian manifolds, $\mathscr{H}_{2 k}$ and $\mathscr{H}_{2 k+1}$ depend not only on $\sigma_{k}$ but also on the Riemannian curvature tensor of the ambient manifolds. Therefore, except for the case that the ambient spaces are the space forms, for which $\mathscr{H}_{2 k}$ and $\mathscr{H}_{2 k+1}$ can be written as linear combinations of $\sigma_{k}$, one cannot express them as pure functions on the principal curvatures of hypersurfaces.

The first attempt in which we succeed is the rigidity on the curvature quotients $\mathscr{H}_{2 k+1} / \mathscr{H}_{2 k}$ in a class of warped product manifolds. These quotients can be viewed as a generalization of the usual mean curvature $H$ since the case $k=0$ corresponds to $H$. We remark that the rigidity on the quotients of $\sigma_{k}$ in a class of warped product manifolds has been considered in [Wu and Xia 2014]. However, as mentioned before, these two kinds of quotients have large differences in general. Many techniques seem to be difficult to apply for the quotients $\mathscr{H}_{2 k+1} / \mathscr{H}_{2 k}$ considered here.

The first main result of this paper is stated as:

Theorem 1.1. Define $\left(M^{n}, \bar{g}\right)$ to be an $n$-dimensional warped product manifold $[0, \bar{r}) \times_{\lambda} N^{n-1}$ whose warped product function satisfies

$$
\lambda \lambda^{\prime \prime}-\left(\lambda^{\prime}\right)^{2} \geq 0 \quad \text { (i.e., } \log \lambda \text { is convex). }
$$

Let $\Sigma^{n-1}$ be a closed star-shaped hypersurface in $M$ such that the generalized Einstein tensor $E_{(k)}$ is semidefinite on $\Sigma$. For any integer $k$ with $0 \leq k<\frac{1}{2}(n-1)$ and $\mathscr{H}_{2 k}$ not vanishing on $\Sigma$, if the curvature quotient $\mathscr{H}_{2 k+1} / \mathscr{H}_{2 k}$ is a constant, then $\Sigma$ is a slice $\left\{r_{0}\right\} \times N$ for some $r_{0} \in[0, \bar{r})$ and the constant is $(n-1-2 k) \log \lambda\left(r_{0}\right)$.

The star-shapedness means that $\Sigma$ can be written as a graph over $N$, alternatively, $\langle\partial / \partial r, v\rangle \geq 0$, where $v$ is the outer normal of $\Sigma$. The method to prove Theorem 1.1 is to apply the maximum principle to an elliptic equation. This method was previously indicated by Montiel [1999] and was used widely in [Alías and Colares 2007; Alías et al. 2012]. 
The condition (1-1) imposed on $M$ only depends on the warped product function $\lambda$, not the fiber manifold $N$. We notice that the condition excludes the usual space forms $\mathbb{R}^{n}, \mathbb{S}_{+}^{n}$ (hemisphere) and $\mathbb{U}^{n}$ (hyperbolic space) in which cases $\lambda \lambda^{\prime \prime}-\left(\lambda^{\prime}\right)^{2}=-1$. For $\mathbb{R}^{n}$, since the quotient $\mathscr{H}_{2 k+1} / \mathscr{H}_{2 k}$ is equal to $\sigma_{2 k+1} / \sigma_{2 k}$, the result still holds; see [Korevaar 1988; Koh 2000]. We will consider the case $\mathbb{S}_{+}^{n}$ and $\mathbb{\boxplus}^{n}$ elsewhere since the proof has a different flavor. We also notice that the condition (1-1) is satisfied by some local space forms such as $[0, \infty) \times e^{r} \mathbb{R}^{n-1}$ or $[0, \infty) \times \cosh r \mathbb{R}^{n-1}$. There are also nonconstant curvature manifolds which satisfy (1-1). A typical example for which the condition (1-1) is satisfied is the so-called Kottler-Schwarzschild spaces $[0, \infty) \times{ }_{\lambda} N(\kappa)$, whose warped product fact $\lambda$ satisfies $\lambda^{\prime}(r)=\sqrt{\kappa+\lambda(r)^{2}-2 m \lambda(r)^{2-n}}$ and $N(\kappa)$ is a closed space form of the constant sectional curvature $\kappa=0$ or -1 . See Appendix for a detailed explanation.

Note that $E_{(1)}^{i j}=R^{i j}-\frac{1}{2} R g^{i j}$ is the Einstein tensor, so that in $k=1$ case, the semidefinite condition of $E_{(1)}$ is just the semidefiniteness of the Einstein tensor. In particular, if $M=\mathbb{R}^{n}$, one readily sees that $-E_{(k)}=\frac{1}{2}(2 k) ! T_{2 k}$, where $T_{2 k}$ is the $2 k$-Newton tensor associated to the hypersurface $\Sigma$, and the seminegative definite condition of $E_{(k)}$ relates to $2 k$-convexity.

In order to extend the above result to noncompact hypersurfaces, we need a generalization of the Omori-Yau maximum principle for the trace-type semi-elliptic operators. The classical Omori-Yau maximum principle is initially stated for the Laplacian $\Delta$. A Riemannian manifold $\Sigma$ is said to satisfy the Omori-Yau maximum principle if for any function $u \in C^{2}(\Sigma)$ with $\sup _{\Sigma} u<+\infty$, there exists a sequence $\left\{p_{i}\right\}_{i \in \mathbb{N}} \subset \Sigma$ such that for each $i$, the following inequalities hold:

$$
u\left(p_{i}\right)>\sup _{\Sigma} u-\frac{1}{i}, \quad|\nabla u|\left(p_{i}\right)<\frac{1}{i}, \quad \Delta u\left(p_{i}\right)<\frac{1}{i} .
$$

This principle was first proved by Omori [1967] and later generalized by Yau [1975] under the condition that the Ricci curvature is bounded from below. It has proved to be very useful in the framework of noncompact manifolds and attracted considerable extending works. For example, it was improved by Chen and Xin [1992] and Ratto, Rigoli and Setti [Ratto et al. 1995] by assuming that the radial curvature decays slower than a certain decreasing function. Recently, the essence of the Omori-Yau maximum principle was captured by Pigola, Rigoli and Setti (see [Pigola et al. 2005, Theorem 1.9]) that the validity of the Omori-Yau maximum principle is assured by the existence of some nonnegative $C^{2}$ function satisfying some appropriate requirements, and thus may not necessarily depend on the curvature bounds. Also, they discussed the generalizations for the trace-type differential operators (see Definition 3.1) which will be used in this paper. For a detailed discussion of the 
sufficient condition to guarantee the Omori-Yau maximum principle for the tracetype differential operators to hold in the warped product manifolds, see [Alías et al. 2013] or Section 3 below.

We have a rigidity result for noncompact hypersurfaces:

Theorem 1.2. Define $\left(M^{n}, \bar{g}\right)$ to be an $n$-dimensional warped product manifold $[0, \bar{r}) \times_{\lambda} N^{n-1}$ whose warped product function satisfies $\lambda \lambda^{\prime \prime}-\left(\lambda^{\prime}\right)^{2} \geq 0$ with equality only at isolated points. Let $\left(\Sigma^{n-1}, g\right)$ be a complete noncompact starshaped hypersurface in $M$, which is contained in a slab $\left[r_{1}, r_{2}\right] \times N, 0 \leq r_{1}<r_{2}<\bar{r}$, such that the generalized Einstein tensor $E_{(k)}$ being semidefinite on $\Sigma$. Assume the Omori-Yau maximum principle holds for the trace-type operator $\operatorname{tr}_{g}\left(-2 E_{(k)} \nabla_{g}^{2}\right)$ on $\Sigma$. For an integer $k$ with $0 \leq k<\frac{1}{2}(n-1)$ and $\mathscr{H}_{2 k}$ not vanishing on $\Sigma$, if the curvature quotient $\mathscr{H}_{2 k+1} / \mathscr{H}_{2 k}$ is a constant, then $\Sigma$ is a slice $\left\{r_{0}\right\} \times N$ for some $r_{0} \in\left[r_{1}, r_{2}\right]$ and the constant is $(n-1-2 k) \log \lambda\left(r_{0}\right)$.

Motivated by the analogous Bernstein type result on the quotient of the usual mean curvatures [Aquino and de Lima 2014], we can establish a corresponding result in our case. More precisely, instead of assuming the curvature quotient $\mathscr{H}_{2 k+1} / \mathscr{H}_{2 k}$ being constant, we can establish the rigidity result via assuming a natural comparison inequality between $\mathscr{H}_{2 k+1} / \mathscr{H}_{2 k}$ and its value on the slices.

Theorem 1.3. Define $\left(M^{n}, \bar{g}\right)$ to be an $n$-dimensional warped product manifold $[0, \bar{r}) \times_{\lambda} N$. Let $\left(\Sigma^{n-1}, g\right)$ be a complete, star-shaped hypersurface in $M$, which is contained in a slab $\left[r_{1}, r_{2}\right] \times N, 0 \leq r_{1}<r_{2}<\bar{r}$, such that the generalized Einstein tensor $E_{(k)}$ is semidefinite on $\Sigma$. Assume that the Omori-Yau maximum principle holds for the trace-type operator $\operatorname{tr}_{g}\left(-2 E_{(k)} \nabla_{g}^{2}\right)$ on $\Sigma$ and that the Gauss-Bonnet curvature $\mathscr{H}_{2 k}$ is bounded by two positive constants, i.e., $0<C_{1} \leq \mathscr{H}_{2 k} \leq C_{2}$. If

$$
\frac{\mathscr{H}_{2 k+1}}{\mathscr{H}_{2 k}} \leq(n-1-2 k) \frac{\lambda^{\prime}(r)}{\lambda(r)} \quad \text { and } \quad\left|\nabla_{g} r\right|_{g} \leq \inf _{\Sigma}\left((n-1-2 k) \frac{\lambda^{\prime}(r)}{\lambda(r)}-\frac{\mathscr{H}_{2 k+1}}{\mathscr{H}_{2 k}}\right) \text {, }
$$

then the hypersurface $\Sigma$ is a slice $\left\{r_{0}\right\} \times M$ for some $r_{0} \in\left[r_{1}, r_{2}\right]$.

We remark that we do not assume the log-convexity of the warped product function for Theorem 1.3.

\section{Preliminaries}

In this section, we first recall the work of [Lovelock 1971] on the generalized Einstein tensors and Gauss-Bonnet curvatures. Throughout this paper, we use the notation $R_{i j k l}, R_{i j}$ and $R$ to indicate the Riemannian 4-tensor, the Ricci tensor in local coordinates and the scalar curvature respectively. We use the metric $g$ to lower or raise an index and adopt the Einstein summation convention: repeated upper and lower indices will automatically be summed unless otherwise noted. 
For an $(n-1)$-dimensional Riemannian manifold $\left(\Sigma^{n-1}, g\right)$, the Einstein tensor $E_{i j}=R_{i j}-\frac{1}{2} R g_{i j}$ is very important in theoretical physics. It is a conversed quantity, i.e.,

$$
\nabla_{j} E_{i}^{j}=0,
$$

where $\nabla$ is the covariant derivative with respect to the metric $g$.

Lovelock [1971] studied the classification of tensors $A$ satisfying

(i) $A^{i j}=A^{j i}$, i.e, $A$ is symmetric.

(ii) $A^{i j}=A^{i j}\left(g, \partial g, \partial^{2} g\right)$.

(iii) $\nabla_{j} A^{i j}=0$, i.e., $A$ is divergence-free.

(iv) $A^{i j}$ is linear in the second derivatives of $g$.

It is clear that the Einstein tensor $E_{i j}$ satisfies all above conditions. Lovelock classified all 2-tensors satisfying (i)-(iii). For an integer $0 \leq k \leq \frac{1}{2}(n-1)$, let us define a 2-tensor $E_{(k)}$ locally by

$$
E_{(k)}^{i j}:=-\frac{1}{2^{k+1}} g^{l j} \delta_{l j_{1} j_{2} \cdots j_{2 k-1} j_{2 k}}^{i i_{1} i_{2} \cdots i_{2 k-1} i_{2 k}} R_{i_{1} i_{2}}^{j_{1} j_{2}} \cdots R_{i_{2 k-1} i_{2 k}}^{{ }^{j}{ }_{2 k-1} j_{2 k}} .
$$

Here the generalized Kronecker delta is defined by

$$
\delta_{i_{1} i_{2} \cdots i_{r}}^{j_{1} j_{2} \cdots j_{r}}=\operatorname{det}\left(\begin{array}{cccc}
\delta_{i_{1}}^{j_{1}} & \delta_{i_{1}}^{j_{2}} & \cdots & \delta_{i_{1}}^{j_{r}} \\
\delta_{i_{2}}^{j_{1}} & \delta_{i_{2}}^{j_{2}} & \cdots & \delta_{i_{2}}^{j_{r}} \\
\vdots & \vdots & \ddots & \vdots \\
\delta_{i_{r}}^{j_{1}} & \delta_{i_{r}}^{j_{2}} & \cdots & \delta_{i_{r}}^{j_{r}}
\end{array}\right) .
$$

One can check that $E_{(k)}$ satisfies (i)-(iii). Lovelock proved that any 2-tensor satisfying (i)-(iii) has the form

$$
\sum_{k} \alpha^{k} E_{(k)}
$$

with certain constants $\alpha^{k}, k \geq 0$. The $E_{(k)}$ are called the generalized Einstein tensors.

For an integer $0 \leq k \leq \frac{1}{2}(n-1)$, the Gauss-Bonnet curvatures $L_{k}$ are defined by

$$
L_{k}:=\frac{1}{2^{k}} \delta_{j_{1} j_{2} \cdots j_{2 k-1} j_{2 k}}^{i_{1} i_{2} \cdots i_{2 k-1} i_{2 k}} R_{i_{1} i_{2}}^{j_{1} j_{2}} \cdots R_{i_{2 k-1} i_{2 k}}^{j_{2 k-1} j_{2 k}}
$$

When $2 k=n-1, L_{k}$ is the Euler density. When $k<\frac{1}{2}(n-1), L_{k}$ is called the dimensional continued Euler density in physics. We set $E_{(0)}=-\frac{1}{2} g$ and $L_{0}=1$. It is clear from the definitions (2-1) and (2-2) that

$$
\operatorname{tr}_{g}\left(E_{(k)}\right):=E_{(k)}^{i j} g_{i j}=-\frac{n-1-2 k}{2} L_{k}
$$


It is easy to see that $\left(E_{(1)}\right)_{i j}=R_{i j}-\frac{1}{2} R g_{i j}$ is the Einstein tensor and $L_{1}=R$ is the scalar curvature. One can also check that

$$
E_{(2)}^{i j}=2 R R^{i j}-4 R^{i s} R_{s}{ }^{j}-4 R_{s l} R^{s i l j}+2 R^{i}{ }_{k l m} R^{j k l m}-\frac{1}{2} g^{i j} L_{2},
$$

and

$$
L_{2}=\frac{1}{4} \delta_{j_{1} j_{2} j_{3} j_{4}}^{i_{1} i_{2} i_{3} i_{4}} R_{i_{1} i_{2}}^{j_{1} j_{2}} R_{i_{3} i_{4}}^{j_{3} j_{4}}=R_{i j s l} R^{i j s l}-4 R_{i j} R^{i j}+R^{2} .
$$

In [Lovelock 1971], the author proved that the first variational formula for the total Gauss-Bonnet curvature functional is given in terms of the generalized Einstein tensor. It was also presented in [Li 1985; Labbi 2008b], although with different notation and formalism. For the convenience of readers, we include a proof here.

Proposition 2.1 [Lovelock 1971]. Let $\left(\Sigma^{n-1}, g\right)$ be a smooth closed manifold. Assume that $g_{t}$ is a variation of $g$ with $\left.\frac{\partial}{\partial t}\right|_{t=0} g_{i j}=v_{i j}$ for a symmetric 2-tensor $v$, then

$$
\left.\frac{d}{d t}\right|_{t=0} \int_{\Sigma_{t}} L_{k} d \mu_{t}=\int_{\Sigma}-E_{(k)}^{i j} v_{i j} d \mu .
$$

In particular, if $\left(\Sigma^{n-1}, g\right)$ is a closed, smooth hypersurface immersed in an $n$ dimensional Riemannian manifold $\left(M^{n}, \bar{g}\right)$ and the variational vector field is given by the outward unit normal $v$, then

$$
\left.\frac{d}{d t}\right|_{t=0} \int_{\Sigma_{t}} L_{k} d \mu_{t}=\int_{\Sigma}-2 E_{(k)}^{i j} h_{i j} d \mu .
$$

where $h_{i j}$ denotes the second fundamental form of $\Sigma$ with respect to $-v$.

Proof. By the simple fact that $\left.\frac{d}{d t}\right|_{t=0} d \mu_{t}=\frac{1}{2} \operatorname{tr}_{g} v d \mu$ and the definition of $L_{k}$, we compute

$$
\begin{aligned}
\left.\frac{d}{d t}\right|_{t=0} \int_{\Sigma_{t}} L_{k} d \mu_{t} & =\left.\int_{\Sigma} \frac{d}{d t}\right|_{t=0} L_{k} d \mu+\int_{\Sigma} \frac{1}{2} L_{k} \operatorname{tr}_{g} v d \mu \\
& =\left.\int_{\Sigma} k P_{(k)_{s l}}^{i j} \frac{d}{d t}\right|_{t=0} R_{i j}{ }^{s l} d \mu+\int_{\Sigma} \frac{1}{2} L_{k} \operatorname{tr}_{g} v d \mu,
\end{aligned}
$$

where the 4-tensor $P_{(k)}$ is given by

$$
P_{(k)}^{s t l j}:=\frac{1}{2^{k}} \delta_{j_{1} j_{2} \cdots j_{2 k-3} j_{2 k-2} j_{2 k-1} j_{2 k}}^{i_{1} i_{2} \cdots i_{2 k-3} i_{2 k-1} s t} R_{i_{1} i_{2}}^{j_{1} j_{2}} \cdots R_{i_{2 k-3} i_{2 k-2}}^{j_{2 k-3} j_{2 k-2}} g^{j_{2 k-1} l} g^{j_{2 k} j},
$$

and

$$
P_{(k)}^{i j}=P_{(k)}^{i j p q} g_{s p} g_{l q} .
$$

We remark that $P_{(k)}$ shares the same symmetry as the Riemann curvature tensor, that is,

$$
P_{(k)}^{s t j l}=-P_{(k)}^{t s j l}=-P_{(k)}^{s t l j}=P_{(k)}^{j l s t} .
$$


Furthermore, by applying the second Bianchi identity of the curvature tensor, one can check that $P_{(k)}$ has the crucial property of being divergence-free (see [Ge et al. 2014, Lemma 2.2] for a proof)

$$
\nabla_{s} P_{(k)}^{s t j l}=0 .
$$

To calculate the first term in (2-6), we recall that if $(\partial / \partial t) g=v$, then the evolution equation of the curvature tensor is given by (see [Chow et al. 2006, Equation (2.66)])

$$
\frac{d}{d t} R_{i j s l}=-\frac{1}{2}\left(\nabla_{i} \nabla_{j} v_{s l}-\nabla_{i} \nabla_{l} v_{s j}-\nabla_{s} \nabla_{j} v_{i l}+\nabla_{s} \nabla_{l} v_{i j}-R_{i j s m} v^{m}{ }_{l}-R_{i j m l} v^{m}{ }_{s}\right) .
$$

Then we use (2-8) and (2-9) to compute that

$$
\begin{aligned}
& \int_{\Sigma} k P_{(k)_{s l}}^{i j}\left(\left.\frac{d}{d t}\right|_{t=0} R_{i j}{ }^{s l}\right) d \mu \\
& =\int_{\Sigma} k P_{(k)_{s l}}^{i j}\left(\frac{1}{2}\left(-\nabla_{i} \nabla_{j} v^{s l}+\nabla_{i} \nabla^{l} v_{j}^{s}+\nabla^{s} \nabla_{j} v_{i}^{l}-\nabla^{s} \nabla^{l} v_{i j}\right)\right. \\
& \left.\quad+\frac{1}{2}\left(R_{i j m}{ }^{l} v^{m s}-R_{i j m}{ }^{s} v^{m l}\right)+\left(-R_{i j p}{ }^{l} v^{s p}-R_{i j q}{ }^{s} v^{l q}\right)\right) d \mu \\
& =-\int_{\Sigma} k P_{(k)_{s l}}^{i j} R_{i j m}{ }^{l} v^{s m} d \mu,
\end{aligned}
$$

where in the last equality we used (2-9), (2-8) and the simple observation that $\left(R_{i j m}{ }^{l} v^{m s}-R_{i j m}{ }^{s} v^{m l}\right)$ and $\left(-R_{i j p}{ }^{l} v^{s p}-R_{i j q}{ }^{s} v^{l q}\right)$ are both antisymmetric with respect to the pair $(s, l)$.

Going back to (2-6), we obtain that

$$
\left.\frac{d}{d t}\right|_{t=0} \int_{\Sigma_{t}} L_{k} d \mu_{t}=\int_{\Sigma}\left(-k P_{(k) l}^{i j s} R_{i j}^{m l}+\frac{1}{2} L_{k} g^{m s}\right) v_{m s} d \mu .
$$

On the other hand, from definitions (2-1), (2-2) and (2-7), it is direct to check that

$$
\begin{aligned}
E_{(k)}^{m s}= & -\frac{1}{2^{k+1}} g^{l s} \delta_{l j_{1} j_{2} \cdots j_{2 k-1} j_{2 k}}^{m i_{1} i_{2} \cdots i_{2 k-1} i_{2 k}} R_{i_{1} i_{2}}{ }^{j_{1} j_{2}} \cdots R_{i_{2 k-1} i_{2 k}}{ }^{j_{2 k-1} j_{2 k}} \\
= & -\frac{1}{2^{k+1}} g^{m s} \delta_{m j_{1} j_{2} \cdots j_{2 k-1} j_{2 k}}^{m i_{1} i_{2} \cdots i_{2 k-1} i_{2 k}} R_{i_{1} i_{2}}{ }^{j_{1} j_{2}} \cdots R_{i_{2 k-1} i_{2 k}}^{j_{2 k-1} j_{2 k}} \\
& \quad-\frac{2 k}{2^{k+1}} g^{i_{1} s} \delta_{i_{1} j_{1} j_{2} \cdots j_{2 k-1} j_{2 k}}^{m i_{1} i_{2} \cdots i_{2 k-1} i_{2 k}} R_{i_{1} i_{2}}{ }^{j_{1} j_{2}} \cdots R_{i_{2 k-1} i_{2 k}}{ }^{j_{2 k-1} j_{2 k}} \\
= & -\frac{1}{2} L_{k} g^{m s}+k P_{(k) l}^{i j s} R_{i j}{ }^{m l} .
\end{aligned}
$$

Hence we complete the proof of (2-4).

In the case that $\Sigma$ is a hypersurface, one only needs to note that $\frac{\partial}{\partial t} g_{i j}=2 h_{i j}$ for the evolving hypersurfaces.

The second aim of this section is to give several simple facts on the warped product manifolds. Let $M^{n}=[0, \bar{r}) \times_{\lambda} N^{n-1}(0<\bar{r} \leq \infty)$ be a warped product 
manifold equipped with a Riemannian metric

$$
\bar{g}=d r^{2}+\lambda(r)^{2} g_{N} .
$$

where $\lambda:[0, \bar{r}) \rightarrow \mathbb{R}$ is a smooth positive function. Let $\Sigma$ be a smooth hypersurface in $(M, \bar{g})$ with induced metric $g$. We denote by $\bar{\nabla}$ and $\nabla$ the covariant derivatives with respect to $\bar{g}$ and $g$ respectively. We define a vector field $X$ on $M$ by

$$
X(r)=\lambda(r) \frac{\partial}{\partial r} .
$$

Let $\left\{e_{1}, \ldots, e_{n-1}\right\}$ be a local frame on $\Sigma$, it is well known that $X$ is a conformal Killing vector field satisfying

$$
\bar{\nabla}_{e_{i}} X(r)=\lambda^{\prime}(r) e_{i}
$$

We denote by $r$ the height function which is obtained by the projection of $\Sigma$ in $M$ onto the first factor $[0, \bar{r})$. Let $\phi(r)$ be a primitive of $\lambda(r)$.

Proposition 2.2. The restriction of $\phi$ on $\Sigma$, still denoted by $\phi$, satisfies

$$
\nabla_{i} \nabla_{j} \phi(r)=\lambda^{\prime}(r) g_{i j}-\langle X, v\rangle h_{i j} .
$$

The height function $r$ on $\Sigma$ satisfies

$$
\nabla_{i} \nabla_{j} r=\frac{\lambda^{\prime}(r)}{\lambda(r)} g_{i j}-\frac{\lambda^{\prime}(r)}{\lambda(r)} \nabla_{i} r \nabla_{j} r-\left\langle\partial_{r}, v\right\rangle h_{i j} .
$$

Consequently, we have

$$
-2 E_{(k)}^{i j} \nabla_{i} \nabla_{j} \phi(r)=(n-1-2 k) \lambda^{\prime}(r) \mathscr{H}_{2 k}-\langle X, v\rangle \mathscr{H}_{2 k+1} .
$$

$$
\text { 5) }-2 E_{(k)}^{i j} \nabla_{i} \nabla_{j} r=(n-1-2 k) \frac{\lambda^{\prime}(r)}{\lambda(r)} \mathscr{H}_{2 k}+\frac{2 \lambda^{\prime}(r)}{\lambda(r)} E_{(k)}^{i j} \nabla_{i} r \nabla_{j} r-\left\langle\partial_{r}, v\right\rangle \mathscr{H}_{2 k+1} \text {. }
$$

Proof. Using (2-11), we have

$$
\begin{aligned}
\nabla_{i} \nabla_{j} \phi(r) & =\bar{\nabla}_{i} \bar{\nabla}_{j} \phi-\langle\bar{\nabla} \phi(r), v\rangle h_{i j}=\bar{\nabla}_{i} X_{j}-\langle X, v\rangle h_{i j} \\
& =\lambda^{\prime}(r) g_{i j}-\langle X, v\rangle h_{i j} .
\end{aligned}
$$

Equation (2-13) follows from (2-12) and

$$
\nabla_{i} \nabla_{j} r=\nabla_{i}\left(\frac{1}{\lambda(r)} \nabla_{j} \phi(r)\right)=\frac{1}{\lambda(r)} \nabla_{i} \nabla_{j} \phi(r)-\frac{\lambda^{\prime}(r)}{\lambda(r)} \nabla_{i} r \nabla_{j} r .
$$

For equations (2-14) and (2-15), we only need to notice that

$$
-2 E_{(k)}^{i j} g_{i j}=(n-1-2 k) L_{k}=(n-1-2 k) \mathscr{H}_{2 k}
$$

and

$$
-2 E_{(k)}^{i j} h_{i j}=\mathscr{H}_{2 k+1} .
$$




\section{3. rigidity for the quotient $\frac{\mathscr{H}_{2 k+1}}{\mathscr{H}_{2 k}}$}

In this section, we prove our main theorems.

Proof of Theorem 1.1. Since $\Sigma$ is compact, there exist points $p_{\min }, p_{\max } \in \Sigma$ such that the height function $r$ attains its maximum and minimum values, i.e.,

$$
\min _{\Sigma} r=r\left(p_{\min }\right), \quad \max _{\Sigma} r=r\left(p_{\max }\right) .
$$

At these points,

$$
\begin{aligned}
& \nabla r\left(p_{\min }\right)=\nabla r\left(p_{\max }\right)=0, \\
& \nabla^{2} r\left(p_{\min }\right) \geq 0, \quad \nabla^{2} r\left(p_{\max }\right) \leq 0 .
\end{aligned}
$$

It follows from (3-1) and the star-shapedness of $\Sigma$ that

$$
\left\langle\partial_{r}, v\right\rangle\left(p_{\min }\right)=\left\langle\partial_{r}, v\right\rangle\left(p_{\max }\right)=1 .
$$

By using (3-1) and (3-3) in (2-15), we obtain

$$
\begin{aligned}
(3-4)-2 E_{(k)}^{i j} \nabla_{i} \nabla_{j} r\left(p_{\min }\right)=(n-1-2 k)(\log \lambda)^{\prime}\left(\min _{\Sigma} r\right) \mathscr{H}_{2 k}\left(p_{\min }\right)-\mathscr{H}_{2 k+1}\left(p_{\min }\right), \\
(3-5)-2 E_{(k)}^{i j} \nabla_{i} \nabla_{j} r\left(p_{\max }\right) \\
=(n-1-2 k)(\log \lambda)^{\prime}\left(\max _{\Sigma} r\right) \mathscr{H}_{2 k}\left(p_{\max }\right)-\mathscr{H}_{2 k+1}\left(p_{\max }\right) .
\end{aligned}
$$

We claim that the quotient $\mathscr{H}_{2 k+1} / \mathscr{H}_{2 k}$ satisfies

$$
\begin{gathered}
\min _{\Sigma}\left(\frac{\mathscr{H}_{2 k+1}}{\mathscr{H}_{2 k}}\right) \leq(n-1-2 k)(\log \lambda)^{\prime}\left(\min _{\Sigma} r\right), \\
(n-1-2 k)(\log \lambda)^{\prime}\left(\max _{\Sigma} r\right) \leq \max _{\Sigma}\left(\frac{\mathscr{H}_{2 k+1}}{\mathscr{H}_{2 k}}\right) .
\end{gathered}
$$

Consider first the case that $-2 E_{(k)}^{i j}$ is positive semidefinite. It follows from (3-2), (3-4) and (3-5) that

$$
\begin{gathered}
(n-1-2 k)(\log \lambda)^{\prime}\left(\min _{\Sigma} r\right) \mathscr{H}_{2 k}\left(p_{\min }\right)-\mathscr{H}_{2 k+1}\left(p_{\min }\right) \geq 0, \\
(n-1-2 k)(\log \lambda)^{\prime}\left(\max _{\Sigma} r\right) \mathscr{H}_{2 k}\left(p_{\max }\right)-\mathscr{H}_{2 k+1}\left(p_{\max }\right) \leq 0 .
\end{gathered}
$$

From the fact that

$$
-2 E_{(k)}^{i j} g_{i j}=(n-1-2 k) \mathscr{H}_{2 k},
$$

together with the assumption that $\mathscr{H}_{2 k}$ is nonvanishing on $\Sigma$, we know that $\mathscr{H}_{2 k}>0$. Hence the claim in this case follows from (3-7) and (3-8) immediately. For the second case that $-2 E_{(k)}^{i j}$ is negative semidefinite, similar argument applies by taking $\mathscr{H}_{2 k}<0$ into account. We finish the proof of the claim. 
Now using the assumption that $\log \lambda$ is convex, we obtain from (3-6) that

$$
\begin{aligned}
\min _{\Sigma}\left(\frac{\mathscr{H}_{2 k+1}}{\mathscr{H}_{2 k}}\right) & \leq(n-1-2 k)(\log \lambda)^{\prime}\left(\min _{\Sigma} r\right) \\
& \leq(n-1-2 k)(\log \lambda)^{\prime}\left(\max _{\Sigma} r\right) \leq \max _{\Sigma}\left(\frac{\mathscr{H}_{2 k+1}}{\mathscr{H}_{2 k}}\right) .
\end{aligned}
$$

Since the quotient $\mathscr{H}_{2 k+1} / \mathscr{H}_{2 k}$ is constant, we have from above that

$$
\frac{\mathscr{H}_{2 k+1}}{\mathscr{H}_{2 k}}=(n-1-2 k)(\log \lambda)^{\prime}\left(\min _{\Sigma} r\right)=(n-1-2 k)(\log \lambda)^{\prime}\left(\max _{\Sigma} r\right),
$$

which yields that $(\log \lambda)^{\prime}(r)$ is a constant function on $\Sigma$. Substituting (3-9) into (2-14), we have

$$
-2 E_{(k)}^{i j} \nabla_{i} \nabla_{j} \phi(r)=\lambda\left(1-\left\langle\partial_{r}, v\right\rangle\right) \mathscr{H}_{2 k+1} .
$$

Notice that $\left\langle\partial_{r}, v\right\rangle \leq 1$ and $\mathscr{H}_{2 k+1}=c \mathscr{H}_{2 k}$ does not change sign on $\Sigma$. Applying the classical maximum principle to the elliptic equation (3-10), we conclude that $\phi(r)$ is a constant function on $\Sigma$. Since $\phi$ is an increasing function with respect to $r$ due to the fact $\phi^{\prime}=\lambda>0$, we conclude that the height function $r$ is a constant function on $\Sigma$, i.e., $\Sigma$ is a slice $\left\{r_{0}\right\} \times N$.

To extend the previous result to noncompact hypersurfaces, we will apply a generalization of the Omori-Yau maximum principle for trace-type differential operators. Consider a Riemannian manifold $(\Sigma, g)$ and a semi-elliptic operator $L=\operatorname{tr}_{g}\left(T \circ \nabla_{g}^{2}\right)$, where $T: T \Sigma \rightarrow T \Sigma$ is a positive semidefinite symmetric tensor. For simplicity we will omit the subscription $g$.

Definition 3.1. We say that the Omori-Yau maximum principle holds on $\Sigma$ for $L$, if for any function $u \in C^{2}(\Sigma)$ with $\sup _{\Sigma} u<+\infty$, there exists a sequence $\left\{p_{i}\right\}_{i \in \mathbb{N}} \subset \Sigma$ such that for each $i$, the following holds:

$$
u\left(p_{i}\right)>\sup _{\Sigma} u-\frac{1}{i}, \quad|\nabla u|\left(p_{i}\right)<\frac{1}{i}, \quad L u\left(p_{i}\right)<\frac{1}{i} .
$$

Since $\inf _{\Sigma} u=-\sup _{\Sigma}(-u)$, the above is equivalent to that for any function $u \in$ $C^{2}(\Sigma)$ with $\inf _{\Sigma} u>-\infty$, there exists a sequence $\left\{p_{i}\right\}_{i \in \mathbb{N}} \subset \Sigma$ such that for each $i$, the following holds:

$$
u\left(p_{i}\right)<\inf _{\Sigma} u+\frac{1}{i}, \quad|\nabla u|\left(p_{i}\right)<\frac{1}{i}, \quad L u\left(p_{i}\right)>-\frac{1}{i} .
$$

Assume the generalized Omori-Yau maximum principle holds for the trace-type operator $L=\operatorname{tr}\left(-2 E_{(k)} \nabla^{2}\right)$, one can prove the analogous result for noncompact hypersurfaces. 
Proof of Theorem 1.2. Due to the same argument as in the proof of Theorem 1.1, we only need to prove the theorem in the case that $-2 E_{(k)}^{i j}$ is positive semidefinite. By the generalized Omori-Yau maximum principle, we have two sequences $\left\{p_{i}\right\}$ and $\left\{q_{i}\right\}$ in $\Sigma$ with properties

(i) $\lim _{i \rightarrow+\infty} \phi\left(r\left(p_{i}\right)\right)=\sup _{\Sigma} \phi(r), \lim _{i \rightarrow+\infty} \phi\left(r\left(q_{i}\right)\right)=\inf _{\Sigma} \phi(r)$;

(ii) $|\nabla \phi(r)|\left(p_{i}\right)=\lambda\left(r\left(p_{i}\right)\right)|\nabla r|\left(p_{i}\right)<\frac{1}{i},|\nabla \phi(r)|\left(q_{i}\right)=\lambda\left(r\left(p_{i}\right)\right)|\nabla r|\left(q_{i}\right)<\frac{1}{i}$;

(iii) $\operatorname{tr}\left(-2 E_{(k)} \nabla^{2} \phi(r)\right)\left(p_{i}\right)<\frac{1}{i}, \operatorname{tr}\left(-2 E_{(k)} \nabla^{2} \phi(r)\right)\left(q_{i}\right)>-\frac{1}{i}$.

Since $\phi(r)$ is strictly increasing due to $\phi^{\prime}(r)=f(r)>0$, we have

and thus

$$
\lim _{i \rightarrow+\infty} r\left(p_{i}\right)=\sup _{\Sigma} r, \quad \lim _{i \rightarrow \infty} r\left(q_{i}\right)=\inf _{\Sigma} r
$$

$$
\lim _{i \rightarrow+\infty}\left\langle\partial_{r}, v\right\rangle\left(p_{i}\right)=\lim _{i \rightarrow+\infty}\left\langle\partial_{r}, v\right\rangle\left(q_{i}\right)=1 .
$$

Using the above facts in (2-14) and letting $i \rightarrow+\infty$, we get

$$
(n-1-2 k)(\log \lambda)^{\prime}\left(\sup _{\Sigma} r\right) \leq \frac{\mathscr{H}_{2 k+1}}{\mathscr{H}_{2 k}} \leq(n-1-2 k)(\log \lambda)^{\prime}\left(\inf _{\Sigma} r\right) .
$$

By the assumption that $(\log \lambda)^{\prime \prime} \geq 0$ with equality only at isolated points, we obtain the desired result that $r$ is constant. That is, $\Sigma$ is a slice $\left\{r_{0}\right\} \times M$.

In the following, we discuss some sufficient condition to guarantee the generalized Omori-Yau maximum principle to hold for $\Sigma$. Inspired by [Pigola et al. 2005], Alías et al. [2013, Theorem 1 and Corollary 3] proved that the Omori-Yau maximum principle holds for a trace-type elliptic operator $L=\operatorname{tr}\left(T \circ \nabla^{2}\right)$ with positive semidefinite $T$ satisfying $\sup _{\Sigma} \operatorname{tr} T<\infty$ on a Riemannian manifold $\Sigma$, provided that the radial sectional curvature (the sectional curvature of the 2-planes containing $\nabla \rho$, where $\rho$ is the distance function on $\Sigma$ from a fixed point in $\Sigma$ ) of $\Sigma$ satisfies the condition

$$
K_{\Sigma}^{\mathrm{rad}}(\nabla \rho, \nabla \rho)>-G(\rho),
$$

where $G:[0,+\infty) \rightarrow \mathbb{R}$ is a smooth function satisfying

$$
G(0)>0, G^{\prime}(t) \geq 0, \int_{0}^{+\infty} \frac{1}{\sqrt{G(t)}}=+\infty, \limsup _{t \rightarrow+\infty} \frac{t G(\sqrt{t})}{G(t)}<+\infty .
$$

A special case for which (3-12) holds is that the sectional curvature of $\Sigma$ is bounded from below (one can choose $G(\rho)=C\left(1+\rho^{2}\right)$, where $C$ is a constant).

In the case of warped product manifolds, Alías et al. gave a detailed discussion of (3-12). More precisely, they proved [ibid., Corollary 4] that for a hypersurface $\Sigma$ in a slab of a warped product manifold $\left[r_{1}, r_{2}\right] \times N,(3-12)$ holds for $L$ with 
positive semidefinite $T$ satisfying $\sup _{\Sigma} \operatorname{tr} T<\infty$, provided that the radial sectional curvature of the fiber manifold $N$ satisfies

$$
K_{N}^{\operatorname{rad}}\left(\nabla^{N} \hat{\rho}, \nabla^{N} \hat{\rho}\right)>-G(\hat{\rho}),
$$

where $\hat{\rho}$ is the distance function on the fiber $N$ from a fixed point in $N$ and $G:[0,+\infty) \rightarrow \mathbb{R}$ is a smooth function satisfying the conditions listed in (3-13), together with $\sup _{\Sigma}\|h\|^{2}<+\infty$ on $\Sigma$. Geometrically, the condition (3-14) means that the radial sectional curvature of the fiber manifold $N$ has a strong quadratic decay at infinity, that is, one can choose $G(\rho)=C\left(1+\rho^{2} \log ^{2}(2+\rho)\right)$ as shown in [Chen and Xin 1992]. In particular, when $N$ has sectional curvature bounded from below or $N$ is compact, (3-14) holds. As a direct result of Theorem 1.2, we have:

Corollary 3.2. Let $\left(M^{n}, \bar{g}\right)$ be as in Theorem 1.2. Assume that the radial sectional curvature of $N$ satisfies (3-14). Let $\Sigma^{n-1}$ be a complete, noncompact star-shaped hypersurface in $M$ which is contained in a slab $\left[r_{1}, r_{2}\right] \times N$ with $\sup _{\Sigma}\|h\|^{2}<+\infty$. Assume $-2 E_{(k)}$ is semidefinite on $\Sigma$ and $\sup _{\Sigma} \mathscr{H}_{2 k}<\infty$ on $\Sigma$. If the quotient $\mathscr{H}_{2 k+1} / \mathscr{H}_{2 k}$ is constant, then the hypersurface is a slice $\left\{r_{0}\right\} \times N$.

Following the argument close to the proof of Theorem 1.2, one may prove the Bernstein-type result in this case.

Proof of Theorem 1.3. By the generalized Omori-Yau maximum principle to the height function $r$, there exists a sequence $\left\{p_{i}\right\} \subset \Sigma$ such that

$$
\lim _{i \rightarrow \infty} r\left(p_{i}\right)=\sup _{\Sigma} r, \quad \lim _{i \rightarrow \infty}|\nabla r|\left(p_{i}\right)=0, \quad \lim _{i \rightarrow \infty} \sup \operatorname{tr}\left(-2 E_{(k)} \nabla^{2} r\right)\left(p_{i}\right) \leq 0 .
$$

It follows from the semidefiniteness of $-2 E_{(k)}$ and the positivity of $\mathscr{H}_{2 k}$ that

$$
0 \leq\left\langle-2 E_{(k)} \nabla r, \nabla r\right\rangle \leq \operatorname{tr}\left(-2 E_{(k)}\right)|\nabla r|^{2} \leq(n-1-2 k) C_{2}|\nabla r|^{2} .
$$

From the fact $\left\langle\partial_{r}, v\right\rangle^{2}=1-|\nabla r|^{2}$, we have

$$
\lim _{i \rightarrow \infty}\left\langle\partial_{r}, v\right\rangle\left(p_{i}\right)=1,
$$

and thus

$$
\lim _{i \rightarrow \infty}\left\langle-2 E_{(k)} \nabla r, \nabla r\right\rangle\left(p_{i}\right)=0 .
$$

Combining all the above facts together into (2-15), we have

$0 \geq \lim _{i \rightarrow \infty} \sup \operatorname{tr}\left(-2 E_{(k)} \nabla^{2} r\right)\left(p_{i}\right) \geq C_{1} \lim _{i \rightarrow \infty}\left((n-1-2 k) \frac{\lambda^{\prime}(r)}{\lambda(r)}-\frac{\mathscr{H}_{2 k+1}}{\mathscr{H}_{2 k}}\right)\left(p_{i}\right) \geq 0$,

so that

$$
\lim _{i \rightarrow \infty}\left((n-1-2 k) \frac{\lambda^{\prime}(r)}{\lambda(r)}-\frac{\mathscr{H}_{2 k+1}}{\mathscr{H}_{2 k}}\right)\left(p_{i}\right)=0 .
$$


From the hypothesis, we have $\inf _{\Sigma}\left((n-1-2 k) \lambda^{\prime}(r) / \lambda(r)-\mathscr{H}_{2 k+1} / \mathscr{H}_{2 k}\right)=0$, and thus $|\nabla r| \equiv 0$ on $\Sigma$, which yields that $\Sigma$ is a slice $\left\{r_{0}\right\} \times M$ for some $r_{0} \in[0, \bar{r})$.

\section{Appendix: Kottler-Schwarzschild manifolds}

The Kottler manifolds, or Kottler-Schwarzschild manifolds, are analogues of the Schwarzschild space in the setting of asymptotically locally hyperbolic manifolds. For $\kappa=1,0$ or -1 , let $(N(\kappa), \hat{g})$ be a closed space form of constant sectional curvature $\kappa$. An $n$-dimensional Kottler-Schwarzschild manifold

$$
P_{\kappa, m}=\left[\rho_{\kappa, m}, \infty\right) \times N(\kappa)
$$

is equipped with the metric

$$
g_{\kappa, m}=\frac{d \rho^{2}}{V_{\kappa, m}^{2}(\rho)}+\rho^{2} \hat{g}, \quad V_{\kappa, m}=\sqrt{\rho^{2}+\kappa-\frac{2 m}{\rho^{n-2}}} .
$$

Let $\rho_{0}:=\rho_{\kappa, m}$ be the largest positive root of

$$
\phi(\rho):=\rho^{2}+\kappa-\frac{2 m}{\rho^{n-2}}=0 .
$$

Remark that in (A-1), in order to have a positive root $\rho_{0}$, if $\kappa=0$ or 1 , the parameter $m$ should be always positive; if $\kappa=-1$, the parameter $m$ can be negative. In fact, in this case, $m \in\left[m_{c},+\infty\right)$ and

$$
m_{c}=-\frac{(n-2)^{(n-2) / 2}}{n^{n / 2}} .
$$

Here the certain critical value $m_{c}$ comes from the following. If $m \leq 0$, one can solve the equation

$$
\phi^{\prime}(\rho)=2 \rho+(n-2) \frac{2 m}{\rho^{n-1}}=0,
$$

to get the root $\rho_{1}=(-(n-2) m)^{1 / n}$. Note the fact that $\phi\left(\rho_{1}\right) \leq 0$, which yields

$$
m \geq-\frac{(n-2)^{(n-2) / 2}}{n^{n / 2}} .
$$

By a change of variable $r=r(\rho)$ with

$$
r^{\prime}(\rho)=\frac{1}{V_{\kappa, m}(\rho)}, \quad r\left(\rho_{\kappa, m}\right)=0,
$$

we can rewrite $P_{\kappa, m}$ as a warped product manifold $P_{\kappa, m}=[0, \infty) \times_{\lambda_{\kappa}} N(\kappa)$ equipped with the metric

$$
g_{\kappa, m}:=\bar{g}:=d r^{2}+\lambda_{\kappa}(r)^{2} \hat{g},
$$

where $\lambda_{\kappa}:[0, \infty) \rightarrow\left[\rho_{\kappa, m}, \infty\right)$ is the inverse of $r(\rho)$, i.e., $\lambda_{\kappa}(r(\rho))=\rho$. 
It is easy to check

$$
\begin{aligned}
& \lambda_{\kappa}^{\prime}(r)=V_{\kappa, m}(\rho)=\sqrt{\kappa+\lambda_{\kappa}(r)^{2}-2 m \lambda_{\kappa}(r)^{2-n}}, \\
& \lambda_{\kappa}^{\prime \prime}(r)=\lambda_{\kappa}(r)+(n-2) m \lambda_{\kappa}(r)^{1-n} .
\end{aligned}
$$

Hence

$$
\lambda_{\kappa} \lambda_{\kappa}^{\prime \prime}-\left(\lambda_{\kappa}^{\prime}\right)^{2}=-\kappa+n m \lambda_{\kappa}^{2-n} .
$$

For the case $\kappa=0, m \geq 0$ and hence $\lambda_{\kappa} \lambda_{\kappa}^{\prime \prime}-\left(\lambda_{\kappa}^{\prime}\right)^{2}=n m \lambda_{\kappa}^{2-n} \geq 0$. For the case $\kappa=-1$, if $m \geq 0$, then $\lambda_{\kappa} \lambda_{\kappa}^{\prime \prime}-\left(\lambda_{\kappa}^{\prime}\right)^{2}=1+n m \lambda_{\kappa}^{2-n}>0$. If

$$
m \in\left[-\frac{(n-2)^{(n-2) / 2}}{n^{n / 2}}, 0\right) \text {, }
$$

then

$$
\begin{aligned}
\lambda_{\kappa} \lambda_{\kappa}^{\prime \prime}-\left(\lambda_{\kappa}^{\prime}\right)^{2} & =1+n m \lambda_{\kappa}^{2-n} \geq 1+n m \rho_{0}^{2-n} \geq 1+n m \rho_{1}^{2-n} \\
& =1+n m(-(n-2) m)^{(2-n) / n}=1-n(n-2)^{(2-n) / n}(-m)^{2 / n} \\
& \geq 1-n(n-2)^{(2-n) / n}\left(\frac{(n-2)^{(n-2) / 2}}{n^{n / 2}}\right)^{2 / n}=0 .
\end{aligned}
$$

As a conclusion, the condition on the log convexity of $\lambda$ holds for the KottlerSchwarzschild manifolds with $\kappa=0$ and -1 . We remark that the log convexity of $\lambda$ does not hold for the Kottler-Schwarzschild manifolds when $\kappa=1$.

\section{Acknowledgments}

Both authors thank Prof. Guofang Wang for his encouragement and constant support. They also thank the referee for a careful reading and critical comments.

\section{References}

[Alexandrov 1956] A. D. Alexandrov, “Теоремы единственности для поверхностей в целом, I", Vestnik Leningrad. Univ. 11:19 (1956), 5-17. Translated as "Uniqueness theorems for surfaces in the large, I" in Amer. Math. Soc. Transl. (2) 21 (1962), 341-354. MR 19,167c Zbl 0122.39601

[Alexandrov 1957] A. D. Alexandrov, “Теоремы единственности для поверхностей в целом, II", Vestnik Leningrad. Univ. 12:7 (1957), 15-44. Translated as "Uniqueness theorems for surfaces in the large, II" in Amer. Math. Soc. Transl. (2) 21 (1962), 354-388. MR 21 \#906 Zbl 0122.39601

[Alexandrov 1958a] A. D. Alexandrov, “Теоремы единственности для поверхностей в целом, III", Vestnik Leningrad. Univ. 13:7 (1958), 14-26. Translated as "Uniqueness theorems for surfaces in the large, III" in Amer. Math. Soc. Transl. (2) 21 (1962), 389-403. MR 21 \#907 Zbl 0269.53024

[Alexandrov 1958b] A. D. Alexandrov, “Теоремы единственности для поверхностей в целом, V", Vestnik Leningrad. Univ. 13:19 (1958), 5-8. Translated as "Uniqueness theorems 
for surfaces in the large, V" in Amer. Math. Soc. Transl. (2) 21 (1962), 412-416. MR 21 \#909 Zbl 0119.16603

[Alexandrov and Volkov 1958] A. D. Alexandrov and Y. A. Volkov, “Теоремы единственности для поверхностей в целом, IV”, Vestnik Leningrad. Univ. 13:13 (1958), 27-34. Translated as "Uniqueness theorems for surfaces in the large, IV" in Amer. Math. Soc. Transl. (2) 21 (1962), 403-411. MR 21 \#908 Zbl 0269.53023

[Alías and Colares 2007] L. J. Alías and A. G. Colares, "Uniqueness of spacelike hypersurfaces with constant higher order mean curvature in generalized Robertson-Walker spacetimes", Math. Proc. Cambridge Philos. Soc. 143:3 (2007), 703-729. MR 2008m:53146 Zbl 1131.53035

[Alías et al. 2012] L. J. Alías, D. Impera, and M. Rigoli, "Spacelike hypersurfaces of constant higher order mean curvature in generalized Robertson-Walker spacetimes", Math. Proc. Cambridge Philos. Soc. 152:2 (2012), 365-383. MR 2887879 Zbl 1241.53051

[Alías et al. 2013] L. J. Alías, D. Impera, and M. Rigoli, "Hypersurfaces of constant higher order mean curvature in warped products", Trans. Amer. Math. Soc. 365:2 (2013), 591-621. MR 2995367 Zbl 1276.53064

[Aquino and de Lima 2014] C. P. Aquino and H. F. de Lima, "On the unicity of complete hypersurfaces immersed in a semi-Riemannian warped product", J. Geom. Anal. 24:2 (2014), 1126-1143. MR 3192308 Zbl 06370417

[Brendle 2013] S. Brendle, "Constant mean curvature surfaces in warped product manifolds", Publ. Math. Inst. Hautes Études Sci. 117 (2013), 247-269. MR 3090261 Zbl 1273.53052

[Brendle and Eichmair 2013] S. Brendle and M. Eichmair, "Isoperimetric and Weingarten surfaces in the Schwarzschild manifold", J. Differential Geom. 94:3 (2013), 387-407. MR 3080487 Zbl 1282.53053

[Chen and Xin 1992] Q. Chen and Y. L. Xin, "A generalized maximum principle and its applications in geometry”, Amer. J. Math. 114:2 (1992), 355-366. MR 93g:53054 Zbl 0776.53032

[Chow et al. 2006] B. Chow, P. Lu, and L. Ni, Hamilton's Ricciflow, Graduate Studies in Mathematics 77, American Mathematical Society, Providence, RI, 2006. MR 2008a:53068 Zbl 1118.53001

[Ge et al. 2014] Y. Ge, G. Wang, and J. Wu, "The GBC mass for asymptotically hyperbolic manifolds", C. R. Math. Acad. Sci. Paris 352:2 (2014), 147-151. MR 3151884 Zbl 1285.53026 arXiv 1306.4233

[Hsiung 1954] C.-C. Hsiung, "Some integral formulas for closed hypersurfaces", Math. Scand. 2 (1954), 286-294. MR 16,849j Zbl 0057.14603

[Koh 2000] S.-E. Koh, "Sphere theorem by means of the ratio of mean curvature functions", Glasg. Math. J. 42:1 (2000), 91-95. MR 2001a:53087 Zbl 0957.53026

[Korevaar 1988] N. J. Korevaar, "Sphere theorems via Alexandrov for constant Weingarten curvature hypersurfaces: appendix to a note of A. Ros", J. Differential Geom. 27:2 (1988), 221-223. MR 89b:53096 Zbl 0638.53052

[Labbi 2008a] M. L. Labbi, “On 2k-minimal submanifolds”, Results Math. 52:3-4 (2008), 323-338. MR 2009m:53160 Zbl 1172.53035

[Labbi 2008b] M. L. Labbi, "Variational properties of the Gauss-Bonnet curvatures", Calc. Var. Partial Differential Equations 32:2 (2008), 175-189. MR 2009a:58013 Zbl 1139.58009

[Lanczos 1938] C. Lanczos, "A remarkable property of the Riemann-Christoffel tensor in four dimensions", Ann. of Math. (2) 39:4 (1938), 842-850. MR 1503440 Zbl 0019.37904

[Li 1985] A. M. Li, "The first and second variations of the Killing invariants", Acta Math. Sinica 28:2 (1985), 154-160. In Chinese. MR 87b:53109b Zbl 0591.58008 
[Liebmann 1899] H. Liebmann, "Eine neue Eigenschaft der Kugel”, Nachr. Ges. Wiss. Göttingen Math.-Phys. Kl. 1899 (1899), 44-55. JFM 30.0557.01

[Lovelock 1971] D. Lovelock, "The Einstein tensor and its generalizations", J. Math. Phys. 12 (1971), 498-501. MR 43 \#1588 Zbl 0213.48801

[Montiel 1999] S. Montiel, "Unicity of constant mean curvature hypersurfaces in some Riemannian manifolds", Indiana Univ. Math. J. 48:2 (1999), 711-748. MR 2001f:53131 Zbl 0973.53048

[Omori 1967] H. Omori, "Isometric immersions of Riemannian manifolds", J. Math. Soc. Japan 19 (1967), 205-214. MR 35 \#6101 Zbl 0154.21501

[Pigola et al. 2005] S. Pigola, M. Rigoli, and A. G. Setti, Maximum principles on Riemannian manifolds and applications, Memoirs of the American Mathematical Society 174:822, American Mathematical Society, Providence, RI, 2005. MR 2006b:53048 Zbl 1075.58017

[Ratto et al. 1995] A. Ratto, M. Rigoli, and A. G. Setti, "On the Omori-Yau maximum principle and its applications to differential equations and geometry", J. Funct. Anal. 134:2 (1995), 486-510. MR 96k:53062 Zbl 0855.58021

[Reilly 1977] R. C. Reilly, "Applications of the Hessian operator in a Riemannian manifold", Indiana Univ. Math. J. 26:3 (1977), 459-472. MR 57 \#13799 Zbl 0391.53019

[Ros 1988] A. Ros, "Compact hypersurfaces with constant scalar curvature and a congruence theorem”, J. Differential Geom. 27:2 (1988), 215-220. MR 89b:53096 Zbl 0638.53051

[Süss 1952] W. Süss, "Über Kennzeichnungen der Kugeln und Affinsphären durch Herrn K.-P. Grotemeyer”, Arch. Math. (Basel) 3 (1952), 311-313. MR 14,789b Zbl 0048.15502

[Wu and Xia 2014] J. Wu and C. Xia, "On rigidity of hypersurfaces with constant curvature functions in warped product manifolds", Ann. Global Anal. Geom. 46:1 (2014), 1-22. MR 3205799 Zbl 06312497

[Yau 1975] S. T. Yau, "Harmonic functions on complete Riemannian manifolds", Comm. Pure Appl. Math. 28 (1975), 201-228. MR 55 \#4042 Zbl 0291.31002

Received December 11, 2013. Revised June 30, 2014.

JIE WU

School of Mathematical Sciences

UNIVERSITY OF SCIENCE AND TECHNOLOGY OF CHINA

HEFEI, 230026

CHINA

wujiewj@ustc.edu.cn

CHAO XIA

SCHOOL of Mathematical SCIENCES

XIAMEN UNIVERSITY

XIAMEN, 361005

CHINA

chaoxia@xmu.edu.cn 


\title{
PACIFIC JOURNAL OF MATHEMATICS
}

\author{
msp.org/pjm
}

Founded in 1951 by E. F. Beckenbach (1906-1982) and F. Wolf (1904-1989)

\section{EDITORS}

Don Blasius (Managing Editor)

Department of Mathematics

University of California

Los Angeles, CA 90095-1555

blasius@math.ucla.edu

\author{
Paul Balmer \\ Department of Mathematics \\ University of California \\ Los Angeles, CA 90095-1555 \\ balmer@math.ucla.edu \\ Robert Finn \\ Department of Mathematics \\ Stanford University \\ Stanford, CA 94305-2125 \\ finn@math.stanford.edu \\ Sorin Popa \\ Department of Mathematics \\ University of California \\ Los Angeles, CA 90095-1555 \\ popa@math.ucla.edu
}

\author{
Vyjayanthi Chari \\ Department of Mathematics \\ University of California \\ Riverside, CA 92521-0135 \\ chari@math.ucr.edu \\ Kefeng Liu \\ Department of Mathematics \\ University of California \\ Los Angeles, CA 90095-1555 \\ liu@math.ucla.edu \\ Jie Qing \\ Department of Mathematics \\ University of California \\ Santa Cruz, CA 95064 \\ qing@ cats.ucsc.edu
}

\section{PRODUCTION}

Silvio Levy, Scientific Editor, production@msp.org

\section{SUPPORTING INSTITUTIONS}

ACADEMIA SINICA, TAIPEI

CALIFORNIA INST. OF TECHNOLOGY

INST. DE MATEMÁTICA PURA E APLICADA

KEIO UNIVERSITY

MATH. SCIENCES RESEARCH INSTITUTE

NEW MEXICO STATE UNIV.

OREGON STATE UNIV.

\author{
STANFORD UNIVERSITY \\ UNIV. OF BRITISH COLUMBIA \\ UNIV. OF CALIFORNIA, BERKELEY \\ UNIV. OF CALIFORNIA, DAVIS \\ UNIV. OF CALIFORNIA, LOS ANGELES \\ UNIV. OF CALIFORNIA, RIVERSIDE \\ UNIV. OF CALIFORNIA, SAN DIEGO \\ UNIV. OF CALIF., SANTA BARBARA
}

\author{
Daryl Cooper \\ Department of Mathematics \\ University of California \\ Santa Barbara, CA 93106-3080 \\ cooper@math.ucsb.edu \\ Jiang-Hua Lu \\ Department of Mathematics \\ The University of Hong Kong \\ Pokfulam Rd., Hong Kong \\ jhlu@maths.hku.hk \\ Paul Yang \\ Department of Mathematics \\ Princeton University \\ Princeton NJ 08544-1000 \\ yang@math.princeton.edu
}

These supporting institutions contribute to the cost of publication of this Journal, but they are not owners or publishers and have no responsibility for its contents or policies.

See inside back cover or msp.org/pjm for submission instructions.

The subscription price for 2015 is US \$420/year for the electronic version, and \$570/year for print and electronic.

Subscriptions, requests for back issues and changes of subscribers address should be sent to Pacific Journal of Mathematics, P.O. Box 4163, Berkeley, CA 94704-0163, U.S.A. The Pacific Journal of Mathematics is indexed by Mathematical Reviews, Zentralblatt MATH, PASCAL CNRS Index, Referativnyi Zhurnal, Current Mathematical Publications and Web of Knowledge (Science Citation Index).

The Pacific Journal of Mathematics (ISSN 0030-8730) at the University of California, c/o Department of Mathematics, 798 Evans Hall \#3840, Berkeley, CA 94720-3840, is published twelve times a year. Periodical rate postage paid at Berkeley, CA 94704, and additional mailing offices. POSTMASTER: send address changes to Pacific Journal of Mathematics, P.O. Box 4163, Berkeley, CA 94704-0163.

PJM peer review and production are managed by EditFLOW ${ }^{\circledR}$ from Mathematical Sciences Publishers.

\section{PUBLISHED BY}

\section{mathematical sciences publishers \\ nonprofit scientific publishing}

http://msp.org/

(C) 2015 Mathematical Sciences Publishers 


\section{PACIFIC JOURNAL OF MATHEMATICS}

Volume $274 \quad$ No. $2 \quad$ April 2015

On Demazure and local Weyl modules for affine hyperalgebras

Angelo Bianchi, Tiago Macedo and Adriano Moura

On curves and polygons with the equiangular chord property

305

TARik Aougab, Xidian Sun, Serge TabachniKov and

YUWEN WANG

The well-posedness of nonlinear Schrödinger equations in Triebel-type 325 spaces

SHAOLEI RU and JiECHENG CHEN

Hypersurfaces with constant curvature quotients in warped product manifolds

JIE WU and CHAO XIA

The first terms in the expansion of the Bergman kernel in higher degrees

MARTin PUCHOL and JiAlin ZHU

Determinant rank of $C^{*}$-algebras

Guinua Gong, HuAXIN Lin and Yifeng XUE

Motion by mixed volume preserving curvature functions near spheres

437

DAVID HARTLEY

Homomorphisms on infinite direct products of groups, rings and monoids

GEORGE M. BERGMAN

The virtual first Betti number of soluble groups

Martin R. BRIDSON and Dessislava H. KochloukOVA 